\title{
A COMPARISON OF FINITE-DIFFERENCE AND FOURIER METHOD CALCULATIONS OF SYNTHETIC SEISMOGRAMS
}

\author{
By C. R. Daudt, L. W. Braile, R. L. Nowack, and C. S. Chiang
}

\begin{abstract}
The Fourier method, the second-order finite-difference method, and a fourthorder implicit finite-difference method have been tested using analytical phase and group velocity calculations, homogeneous velocity model calculations for disperson analysis, two-dimensional layered-interface calculations, comparisons with the Cagniard-de Hoop method, and calculations for a laterally heterogeneous model. Group velocity rather than phase velocity dispersion calculations are shown to be a more useful aid in predicting the frequency-dependent travel-time errors resulting from grid dispersion, and in establishing criteria for estimating equivalent accuracy between discrete grid methods. Comparison of the Fourier method with the Cagniard-de Hoop method showed that the Fourier method produced accurate seismic traces for a planar interface model even when a relatively coarse grid calculation was used. Computations using an IBM 3083 showed that Fourier method calculations using fourth-order time derivatives can be performed using as little as one-fourth the CPU time of an equivalent secondorder finite-difference calculation. The Fourier method required a factor of 20 less computer storage than the equivalent second-order finite-difference calculation. The fourth-order finite-difference method required two-thirds the CPU time and a factor of 4 less computer storage than the second-order calculation. For comparison purposes, equivalent runs were determined by allowing a group velocity error tolerance of $\mathbf{2 . 5}$ per cent numerical dispersion for the maximum seismic frequency in each calculation. The Fourier method was also applied to a laterally heterogeneous model consisting of random velocity variations in the lower half-space. Seismograms for the random velocity model resulted in anticipated variations in amplitude with distance, particularly for refracted phases.
\end{abstract}

\section{INTRODUCTION}

Major advancements in the development of finite-difference and related synthetic seismogram modeling techniques over the past several years have significantly increased the ability to model heterogeneous structures of geophysical interest. The Fourier or pseudo-spectral method, introduced to the geophysical community by Kosloff and Baysal (1982), and higher-order finite-difference methods, for example, the fourth-order implicit method by Fairweather and Mitchell (1965), have been demonstrated to yield equivalent results to the standard second-order explicit finitedifference method while substantially reducing the use of computing resources (Daudt, 1983; Bayliss et al., 1986; Dablain, 1986; Fornberg, 1987). Traditionally, modeling techniques have been limited to lateral variations in two-dimensional media due to practical computing limitations. Recently, however, the Fourier method has been utilized in the development of three-dimensional modeling techniques (Johnson, 1984; Reshef et al., 1988a, b). These efficient methods have facilitated the quantitative investigation of lateral velocity and structural variations for models of full crustal scale and larger, beyond the realm of other modeling methods. 
Both Fourier and high-order finite-difference methods use a much coarser grid than the second-order finite-difference counterpart, as few as two grid points per shortest seismic wavelength for the Fourier method and four or five points per wavelength for fourth or higher-order finite-difference methods. In contrast, the second-order explicit finite-difference method requires 10 or more grid points per wavelength (Alford et al., 1974). The accuracies of the resulting calculations, however, are dependent upon parameters other than grid coarseness and are not clearly understood for heterogeneous models. Several studies have utilized phase velocity numerical dispersion curves as a measure of accuracy for homogeneous models (Alford et al., 1974; Emerman et al., 1982; Daudt, 1983; Fornberg, 1987). Trefethen (1982) utilized both phase and group velocity calculations, noting that the latter provides a more accurate measure of error in travel time.

Recently, attention has been focused on the ability of each method to accurately model inhomogeneous media (Marfurt, 1984; Fornberg, 1987, 1988). Reshef and Kosloff (1985) observed noise from diffractions from the grid points along a sloping fluid-solid interface. Trefethen (1982) discusses the occurrence of parasitic waveforms caused at interfaces from even slight dispersion and warns that boundaries can still introduce instability. Fornberg (1987) performed some comparisons between the Fourier method and fourth- and second-order explicit finite-difference methods with heterogeneous models and noted the Fourier method performed far better than present theory would suggest in many situations. In a more recent paper, Fornberg (1988) described a technique by which Fourier method calculations of heterogeneous models can be improved by calculating derivatives of timeinvariant parameters separately using a smoothly varying grid and mapping the derivatives with the coarsely calculated time-dependent variables.

In the present paper, we provide a further evaluation of finite-difference and Fourier method calculations for homogeneous and selected heterogeneous models using the acoustic-wave equation. The evaluation includes the following:

1. Quantitative prediction of errors resulting from dispersion. The dispersion calculations presented here are based on wave propagation in homogeneous models, but provide a preliminary measure of accuracy for comparison between different methods. Phase and group velocity curves are presented for explicit finite difference, fourth-order implicit finite-difference and Fourier (both second and fourth-order time derivative) methods.

2. Criteria for establishing equivalent-accuracy estimates. The group velocity calculations are utilized to provide a quantitative prediction of errors resulting from dispersion for given model and source parameters. The error estimates are subsequently used to establish equivalent-accuracy criteria for comparing finite-difference and Fourier methods.

3. Comparison of Fourier and Cagniard-de Hoop calculations. A trace by trace comparison of Fourier and Cagniard-de Hoop methods is shown for a model consisting of two homogeneous half-spaces separated by a planar interface.

4. Comparison of Fourier and finite-difference methods. A trace by trace comparison and summary of computer requirements are presented for a model consisting of a free surface and layer over a half-space.

5. Application of the Fourier method to a laterally heterogeneous model. Seismograms are presented for a layer over a half-space model consisting of random velocity variations in the basement, utilizing the Fourier method. 


\section{ANALYTICAL BACKground: Finite-DiffEREnCE METHOdS}

Consider the homogeneous two-dimensional acoustic-wave equation

$$
\frac{\partial^{2} U}{\partial t^{2}}=c^{2}\left(\frac{\partial^{2} U}{\partial x^{2}}+\frac{\partial^{2} U}{\partial z^{2}}\right)
$$

where $U(x, z, t)$ is the acoustic potential and $c$ is the velocity of the medium. Equations (2) and (3) below show second-order explicit and fourth-order implicit finite-difference representations for (1), respectively, the latter obtained from Fairweather and Mitchell (1965).

$$
\begin{gathered}
U_{i j}^{n+1}=\left(p^{2}\left(D_{x}{ }^{2}+D_{z}{ }^{2}\right)+2\right) U_{i j}^{n}-U_{i j}^{n-1} \\
\left(1+\frac{1-p^{2}}{12} D_{x}^{2}\right)\left(1+\frac{1-p^{2}}{12} D_{z}{ }^{2}\right) U_{i j}^{n+1} \\
=\left(1+\frac{1-p^{2}}{12} D_{x}^{2}\right)\left(1+\frac{1-p^{2}}{12} D_{z}^{2}\right)\left(2 U_{i j}^{n}-U_{i j}^{n-1}\right) \\
+p^{2}\left(\left(D_{x}{ }^{2}+D_{z}{ }^{2}\right)+\frac{1}{6} D_{x}{ }^{2} D_{z}{ }^{2}\right) U_{i j}^{n}
\end{gathered}
$$

where

$$
p=c \frac{\Delta t}{\Delta x}
$$

Subscripts $i$ and $j$ and superscript $n$ represent the $x, z$ and time coordinates, respectively, for a discrete grid of uniform spacing, $\Delta x$ and time step size, $\Delta t$, and the delta operators repesent the second-order difference operators

$$
\begin{aligned}
& D_{x}^{2} U_{i j}^{n}=\frac{U_{i+1 j}^{n}-2 U_{i j}^{n}+U_{i-1 j}^{n}}{\Delta x^{2}} \\
& D_{z}^{2} U_{i j}^{n}=\frac{U_{i j+1}^{n}-2 U_{i j}^{n}+U_{i j-1}^{n}}{\Delta z^{2}}
\end{aligned}
$$

Notice the occurrence of the difference operators on the left hand side of the implicit representation (3) resulting in multiple unknown terms. A schematic representation of the grid points used in (2) and (3) is shown in Figure 1.

Dispersion curves for (2) and (3), shown in Figures 2 and 3, respectively, provide a preliminary means of comparing the two finite-difference representations. The familiar phase velocity curves of Figure 2 are derived from Alford et al. (1974) and those of Figure 3 are from Daudt (1983). We have also calculated group velocity curves, shown in the same figures. The curves illustrate numerical dispersion as a function of the ratio of the smallest seismic wavelength to the grid spacing (horizontal axis), $p$ (curves in Figs. $2 \mathrm{~A}$ and $3 \mathrm{~A}$ ) and propagation angle with respect to the grid (curves in Figs. 2B and $3 \mathrm{~B}$ ). 


\section{Explicit Finite Difference}

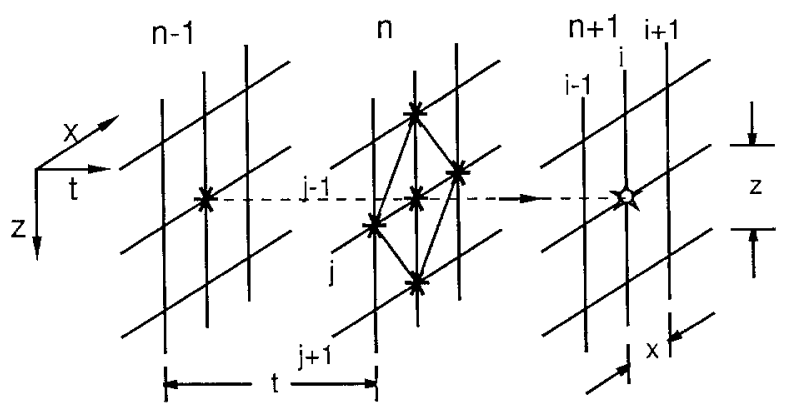

Implicit Finite Difference

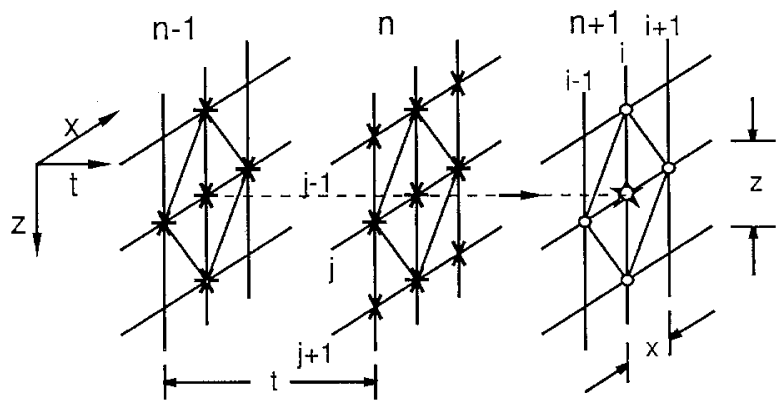

FIG. 1. Comparison of grid points used between explicit and implicit finite-difference forms. The stars represent the unknown terms being calculated and the asterisks represent grid points used which have known values from previous time step calculations or initial conditions. The open circles in the implicit schematic represent surrounding grid points used that are also initially unknown, but are obtainable from boundary conditions and significantly enhance the accuracy of the calculation.

Dispersion for the second-order explicit finite-difference method is commonly considered negligible if the grid density is at least ten points per wavelength $(1 / \mathrm{G}$ $=0.1$ in Fig. 2) and $p$ is about 0.7 (Alford et al., 1974). The curves for Figure 3, however, are much flatter, suggesting an equivalent calculation of the same level of numerical dispersion can be obtained with the Fairweather and Mitchell formulation (3) using only four or five points per wavelength, and again using $p \approx 0.7$. Therefore, based on a comparison of the curves in Figures 2 and 3, the fourth-order formulation is more efficient in grid storage by a factor of 4 for a two-dimensional model and uses half as many time steps.

The dispersion curves (Figs. 2 and 3) indicate another significant but generally overlooked phenomenon, that group velocity dispersion is more sensitive to grid spacing than phase velocity dispersion. This observation has been previously discussed by Trefethen (1982). Both sets of curves indicate less dispersion for the fourth-order implicit method. The group velocity curves that we calculated, however, provide a means of predicting dispersion resulting from coarse grid parameters. Later in this paper, we present experimental tests for dispersion prediction.

A general form of the acoustic-wave equation in (1) allows for heterogeneous media,

$$
\frac{\partial^{2} U}{\partial t^{2}}=\rho c^{2}\left(\frac{\partial}{\partial x}\left(\frac{1}{\rho} \frac{\partial U}{\partial x}\right)+\frac{\partial}{\partial x}\left(\frac{1}{\rho} \frac{\partial U}{\partial z}\right)\right),
$$




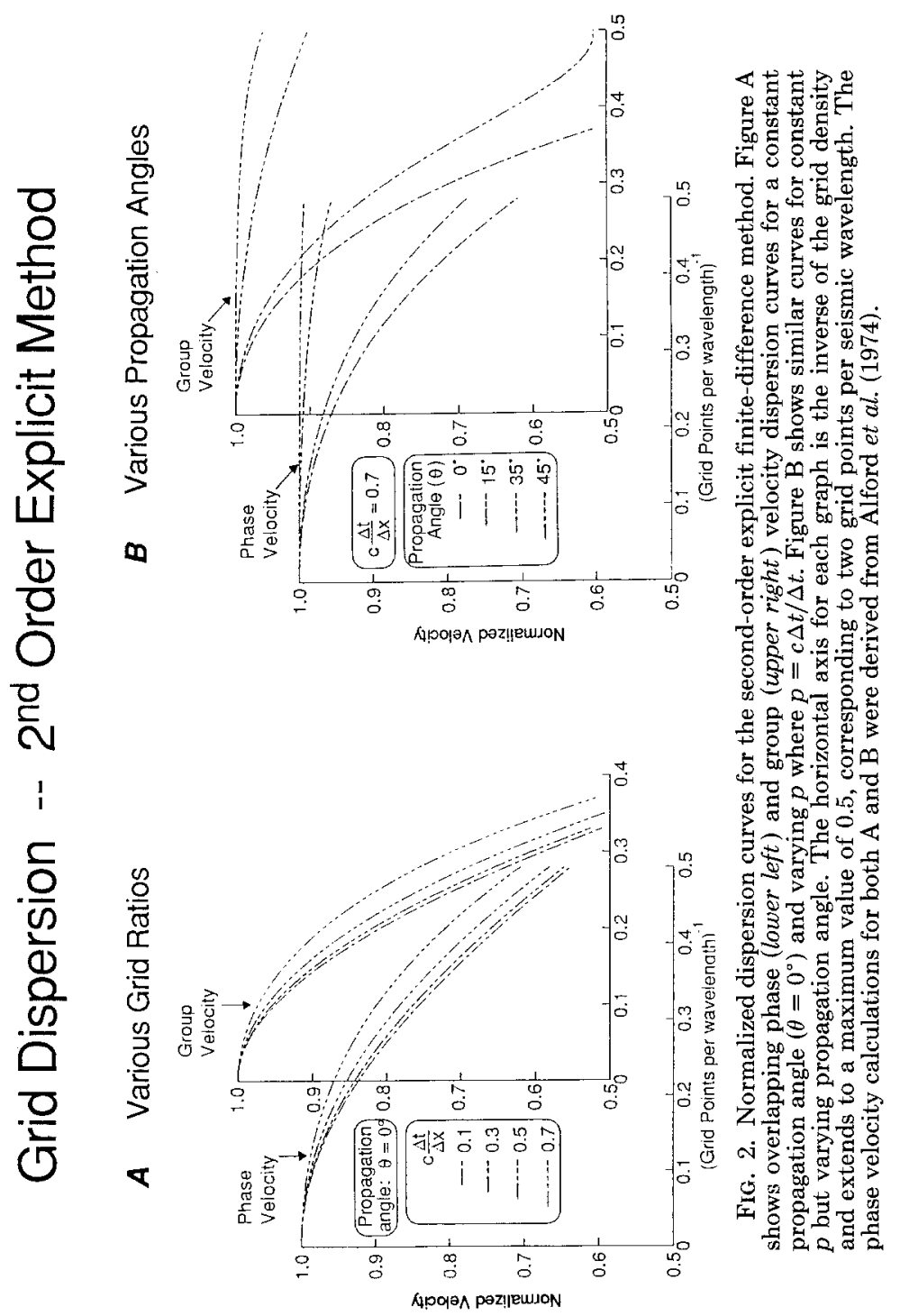




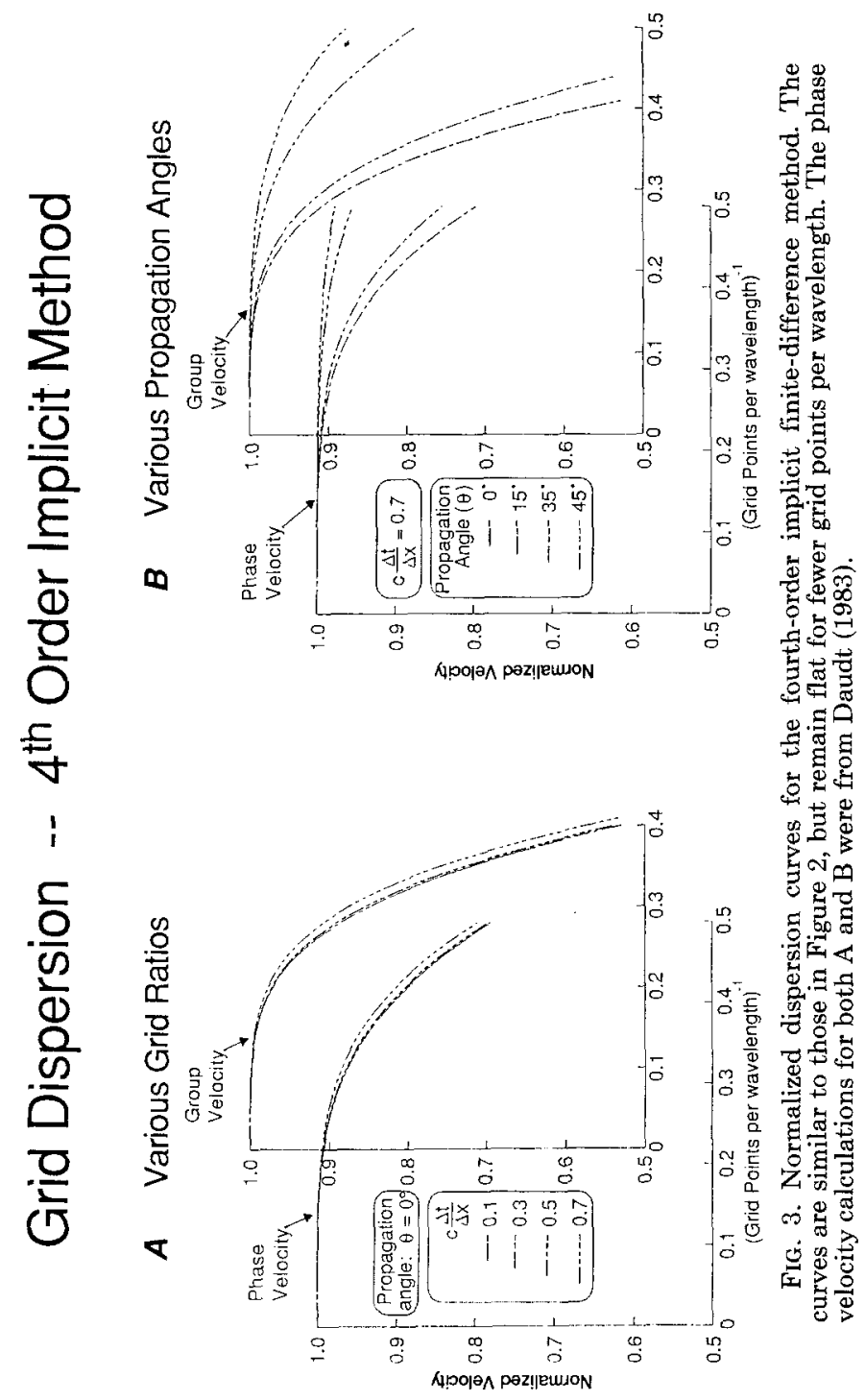


where the velocity, $c(x, z)$ and density, $\rho(x, z)$ of the medium are functions of spatial coordinates. Fairweather and Mitchell's equation has been generalized to follow (5) (modified from McKee, 1973),

$$
\begin{aligned}
\left(1+\frac{\rho D_{x}^{2} \rho^{-1}-p^{2} D_{x}^{2}}{12}\right)\left(1+\frac{\rho D_{z}^{2} \rho^{-1}-p^{2} D_{z}^{2}}{12}\right) U_{i j}^{n+1} & \\
= & \left(1+\frac{\rho D_{x}^{2} \rho^{-1}-p^{2} D_{x}^{2}}{12}\right)\left(1+\frac{\rho D_{z}{ }^{2} \rho^{-1}-p^{2} D_{z}^{2}}{12}\right)\left(2 U_{i j}^{n}-U_{i j}^{n-1}\right) \\
& +p^{2}\left(D_{x}{ }^{2}+D_{z}{ }^{2}+\frac{1}{12}\left(\left(\rho D_{z}{ }^{2} \rho^{-1}\right) D_{x}{ }^{2}+\left(\rho D_{x}{ }^{2} \rho^{-1}\right) D_{z}{ }^{2}\right)\right) U_{i j}^{n} .
\end{aligned}
$$

Equation (6) can be solved using an alternating direction implicit (ADI) approach (Fairweather and Mitchell, 1965) and is the basis for a heterogeneous calculation presented later in this paper.

\section{Analytical Background: The Fourier Method}

Like the second-order finite-difference method of (2), the Fourier method commonly uses a second-order or fourth-order difference approximation for the time derivative. The spatial derivatives, however, are each solved by multiplication in the wavenumber domain. Thus,

$$
\begin{aligned}
\frac{\partial U}{\partial x} & =\frac{\partial}{\partial x} \frac{1}{N} \sum_{0}^{N-1} \hat{U}(k) e^{2 \pi i k x / N} \\
& =\frac{1}{N} \sum_{0}^{N-1} \frac{2 \pi i k}{N} \hat{U}(k) e^{2 \pi i k x / N}
\end{aligned}
$$

where,

$$
\hat{U}(k)=\sum_{0}^{N-1} U(x) e^{-2 \pi i k x / N}
$$

is the Fourier transform of $U(x)$. The spatial derivatives result in negligible dispersion and require only that the grid spacing be adequate to avoid problems associated with aliasing. Tadmor (1986) showed that the dispersion error in spatial derivatives decays to zero at an exponential rate. Therefore, any noticeable dispersion produced by the Fourier method is anticipated to derive from errors in the time derivative alone.

The phase and group velocity curves of Figure 4 indicate that the Fourier method surpasses the fourth-order implicit method in accuracy under certain conditions. The phase velocity curves in Figure 4A, which were based on a second-order time derivative calculation, were derived from Kosloff and Baysal (1982). We calculated the phase velocity curves in Figure $4 \mathrm{~B}$ using fourth-order time derivative terms and also the group velocity curves in both Figures $4 \mathrm{~A}$ and $4 \mathrm{~B}$. The curves show significant reverse dispersion for large values of $p$. However, if $p \leqq 0.1$, the dispersion is negligible up to Nyquist conditions, i.e., as few as two points per wavelength in space $(1 / G=0.5)$, even for the second-order time derivative calculations. Typically, 


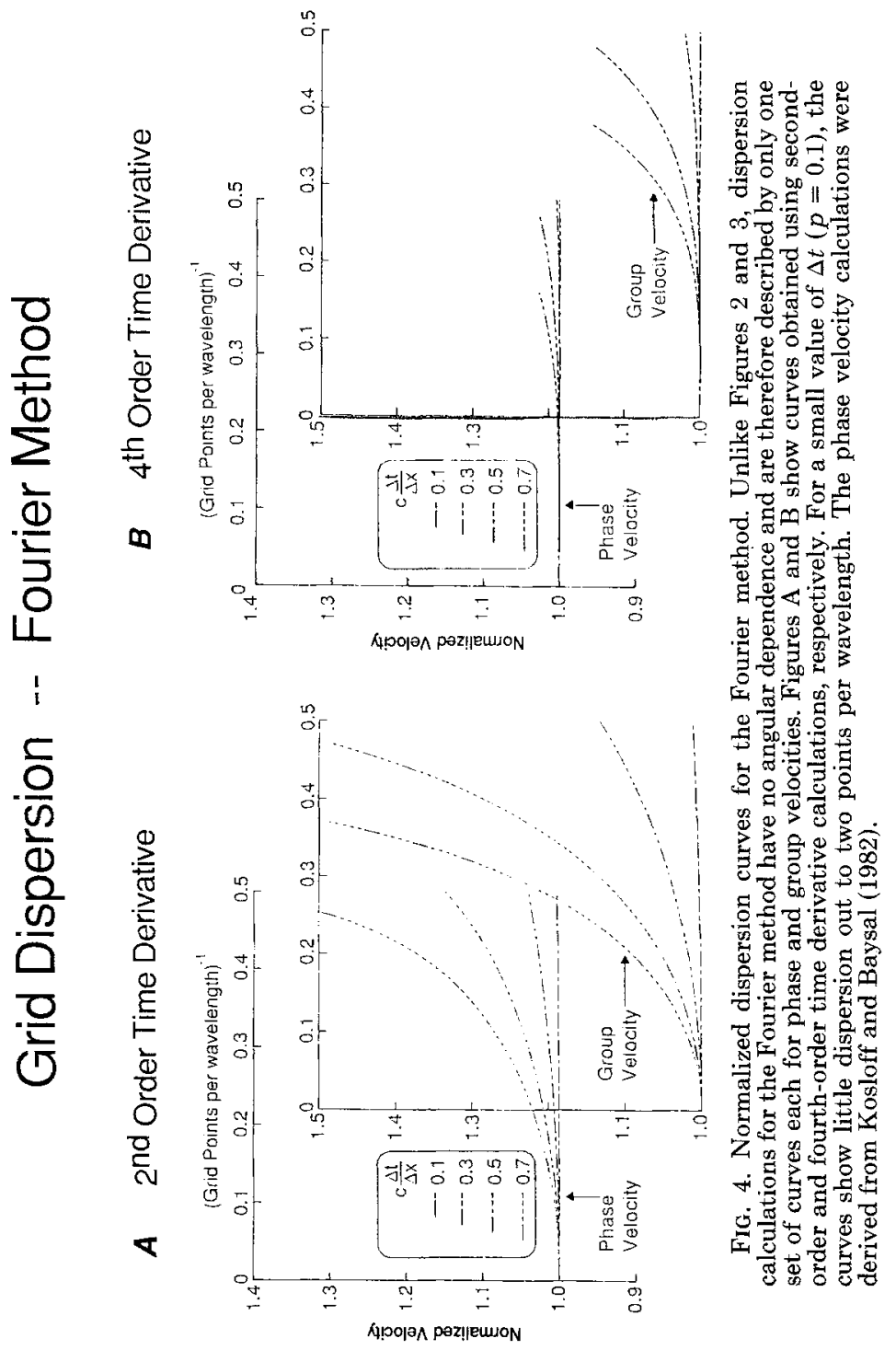


$p$ is allowed to approach 0.2 for the curves in Figure 4A (Kosloff $e t$ al., 1984), and somewhat larger for the fourth-order time derivative curves of Figure $4 \mathrm{~B}$. Since dispersion for the Fourier method is independent of grid spacing, it is also independent of propagation angle through the model. The Fourier method is therefore isotropic, unlike finite-difference methods.

The Fourier method as implemented above is therefore extremely efficient in grid requirements, better than the second-order explicit method by a factor of 25 for a two-dimensional model, but less efficient in total number of required time iterations. Even the time iteration efficiency is improved, however, by using the fourth-order time derivative terms, as recommended by Dablain (1986). Tal-ezer (1986) presents a modification that solves time derivatives as well as spatial derivatives using Fourier transforms.

As with other finite-difference methods, the dispersion curves of Figure 4 indicate that group velocity dispersion is more sensitive to grid spacing than phase velocity dispersion. The curves for group velocity dispersion are in many respects a better measure of accuracy for a discrete grid method, although most geophysical studies have focused primarily on phase velocity calculations. In the following section, we will show how the group velocity curves in Figure $4 \mathrm{~A}$ can be used to predict the effects of dispersion resulting from coarse grid parameters.

\section{EXPERIMENTAL COMPARISONS}

Computer programs have been implemented for second-order explicit and fourthorder implicit finite-difference methods and for the Fourier method. We have written computer codes for both the elastic- and acoustic-wave equations for the explicit and Fourier methods. The comparisons presented here were all based on the acoustic-wave equation. Absorbing boundary conditions have been effectively implemented using methods from Cerjan et al. (1985) and Sochacki et al. (1987). The Cerjan et al. method was chosen for the calculations presented in this paper. The source functions used here were the asymmetric and symmetric waveforms in Figure 5 (Ricker, 1977). In each calculation, the source function was applied at a single grid location over enough time steps to represent the waveform (generally 25 to 40 time steps). The maximum frequency of the wavelets, used for determining grid and time step spacing requirements for a given model, was arbitrarily chosen to be three times the peak frequency, where the amplitude is $25 \mathrm{~dB}$ below peak level for the asymmetric wavelet and $50 \mathrm{~dB}$ below peak level for the symmetric wavelet.

Calculations were either computed on one of several UNIX-based computers or an IBM 3083 computer. Models tested include one-dimensional free-space propagation through a uniform medium, two-dimensional propagation across a simple welded interface and two-dimensional propagation through a uniform layer with a free surface over a uniform half-space, from which equivalent-accuracy criteria were established and comparisons were performed between Fourier, Cagniard-de Hoop, and finite-difference calculations. A laterally heterogeneous model was calculated, which propagated waves through a layer over a half-space with random velocity variations.

Dispersion is readily observed in one-dimensional calculations of homogeneous models, such as the Fourier method calculation illustrated in Figure 6. Seismograms located at the source and at four other distances are shown for a $5.0 \mathrm{~km} / \mathrm{sec}$ medium corresponding to $0,5,10,15$, and $20 \mathrm{sec}$, elapsed travel time. The source wavelet, an asymmetric, $1.0 \mathrm{~Hz}$ peak frequency Ricker wavelet was propagated across 1024 


\section{Asymmetric Wavelet Frequency Spectrum}
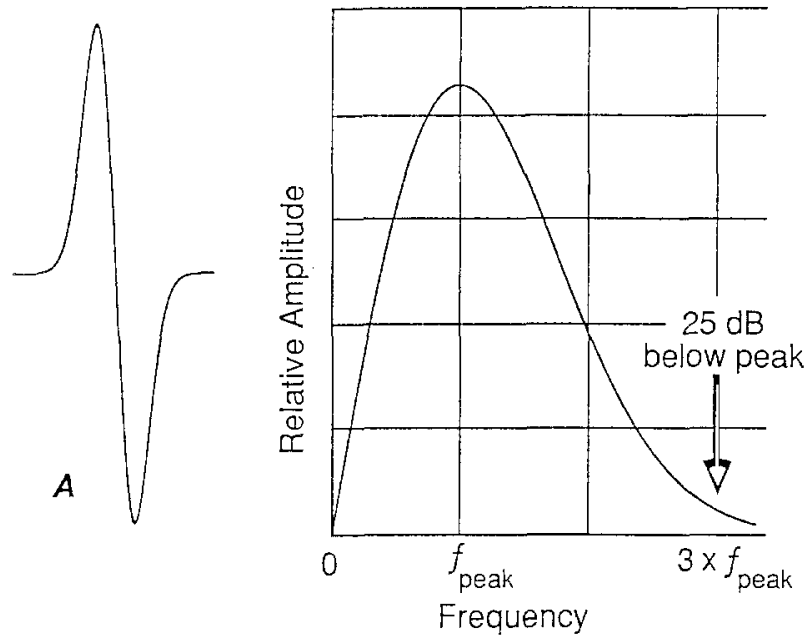

Ricker Wavelet Frequency Spectrum
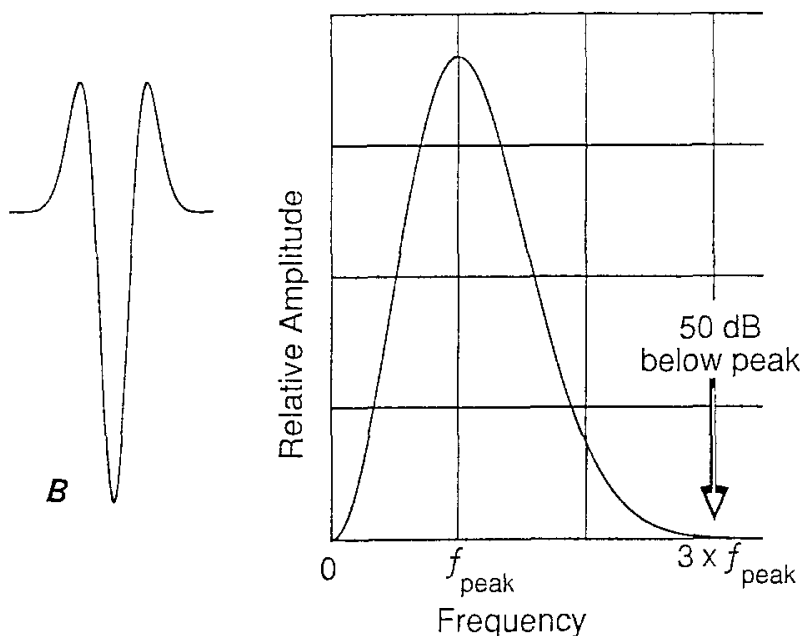

FIG. 5. Frequency spectrums for first-derivative (asymmetric) and second-derivative (symmetric) Gaussian or Ricker velocity-type wavelets (Figures A and B, respectively). Finite-difference and Fourier method calculations presented in this paper were gridded for frequencies up to three times the peak frequency, where the amplitude is $25 \mathrm{~dB}$ below peak amplitude for Figure $\mathrm{A}$ and $50 \mathrm{~dB}$ below peak amplitude for Figure $B$.

grid points and over 600 time steps with grid and time intervals of $416.7 \mathrm{~m}$ and $41.67 \mathrm{msec}$, respectively. The method used was the one-dimensional Fourier method similar to that used by Dablain (1986) with a second-order time derivative calculation. Note that reverse dispersion of the higher frequencies, which is primarily due to the explicit calculation of the time derivative, progresses with each later seismogram. The vertical dashed line through each seismogram shows the correct horizontal placement of the wavelet center in absence of dispersion. The amount of dispersion can be reduced either be decreasing the size of the time step and/or, as is done for most calculations presented in this paper, implementing fourth-order time derivative calculations.

Below each seismogram are five sine waveforms representing five frequencies 
Numerical Dispersion as Predicted from Group Velocity Calculations

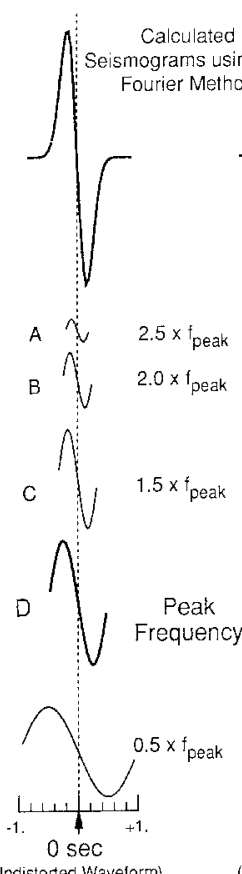

Undistorted Wavelorm
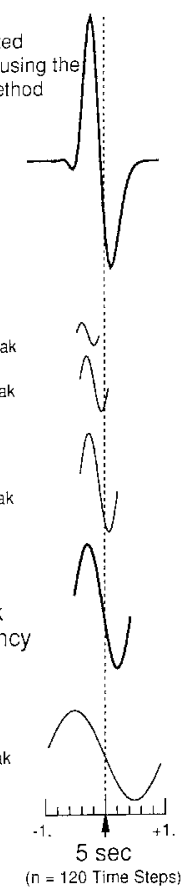
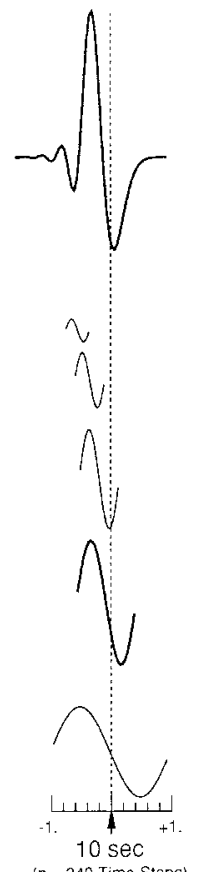

( $n=240$ Time Steps)

Elapsed Time
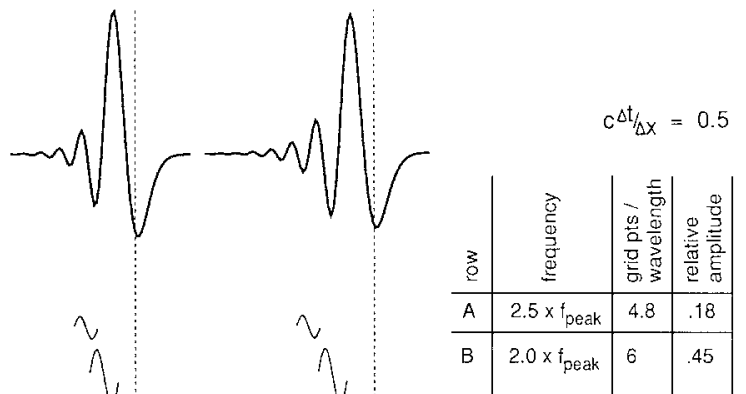

FIG. 6. Numerical dispersion as predicted from group velocity calculations. The top row of curves illustrate the effects of numerical dispersion of a $1.0 \mathrm{~Hz}$ peak frequency source wavelet with time for receivers up to $100 \mathrm{~km}$ ( $20 \mathrm{sec}$ travel time in a $5.0 \mathrm{~km} / \mathrm{sec}$ homogeneous medium) from the source. The calculation method was a one-dimensional implementation of the Fourier method using a second-order time derivative calculation. Grid parameters were $416.7 \mathrm{~m}$ and $41.67 \mathrm{~ms}$ for $\Delta x$ and $\Delta t$, respectively, $p=$ $c \Delta t / \Delta x=0.5,600$ time step iterations and 1024 grid points. The vertical dashed line through each curve indicates the expected location of the center of the waveform when dispersion is absent. Below each seismogram are five sine waves representing five different frequencies contained within the source function. The vertical size of each sine wave is plotted proportional to the relative amplitude obtained from the frequency spectrum for the asymmetric wavelet in Figure $5 \mathrm{~A}$. The relative amplitudes for each sine wave are also indicated by a fractional amplitude factor and scale on the left side of the figure. The frequency, grid density, and relative amplitude for each sine wave are summarized in the table. More importantly, the horizontal position of each sine wave is precisely plotted based on calculations from the group velocity dispersion curves of Figure $4 \mathrm{~A}$. The horizontal locations of the sine waves therefore serve as schematic prediction of numerical dispersion.

contained within the source wavelet. The relative vertical size of the waveforms are in accordance with the amplitude spectrum of the asymmetric source wavelet of Figure 5A. The horizontal position of each waveform is based on the group velocity calculations from Figure 4A for the curve $p=c \Delta t / \Delta x=0.5$. Schematically, Figure 6 illustrates the utility of group velocity dispersion calculations in determining the resulting distortion of the propagated waveform, and in establishing equivalent accuracy criteria for comparing discrete grid methods.

The predictable aspect of dispersion is further illustrated more precisely in Figure 7. Seismograms for three different Fourier calculations are displayed for the receiver at $100 \mathrm{~km}$ in Figure 6, except that the asymmetric source function has been replaced in each of the three calculations by different monochromatic (sinusoidal) source functions. As in Figure 6, the vertical line represents the correct horizontal placement of the wavelet center in absence of dispersion. Each wave packet is positioned horizontally based on its calculated arrival time. The horizontal placement of each arrow gives the predicted location of the center of the corresponding wave packet 


\section{Predicted \& Calculated Numerical}

Dispersion for Three Frequencies

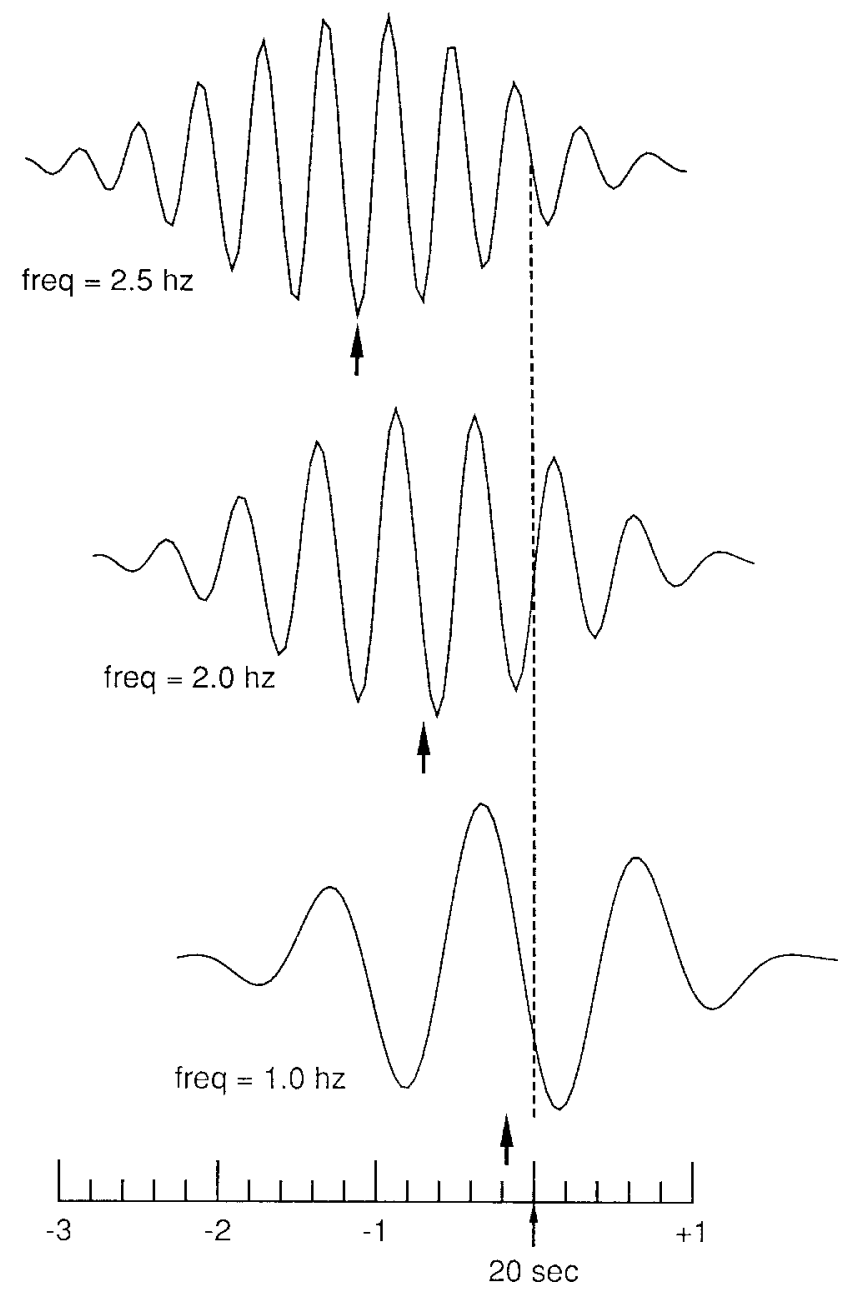

FIG. 7. Seismograms for three different Fourier calculations are displayed for the $100 \mathrm{~km}$ receiver in Figure $6(20 \mathrm{sec})$, except that the asymmetric source function has been replaced in each of the three calculations by a different monochromatic (sinusoidal) source function. As in Figure 6 , the vertical line represents the correct horizontal placement of the wavelet center in absence of dispersion. Each wave packet is accurately positioned horizontally based upon its calculated arrival time. The horizontal placement of each arrow precisely depicts the predicted location of the center of the corresponding wave packet based on group velocity calculations from Figure $4 \mathrm{~A}$. The visual alignment between the arrows and the actual wave packet are verification for Trefethen's (1982) observation that group velocity, rather than phase velocity, better predicts the effects of numerical dispersion in discrete grid methods.

based on group velocity calculations from Figure 4A. The visual alignment between the arrows and the actual wave packet provides verification for Trefethen's (1982) observation that group velocity, rather than phase velocity, better predicts the effects of numerical dispersion in discrete grid methods.

While the one-dimensional homogeneous calculations above were designed to illustrate the effects of dispersion, other effects or limitations of a discrete grid method require analysis of two-dimensional heterogeneous models. We begin with an elementary but fundamentally important comparison of the Fourier method with 
the Cagniard-de Hoop method for a welded interface. The model used in the comparison consisted of a $2.5 \mathrm{~km} / \mathrm{sec}$ half-space above a $4.0 \mathrm{~km} / \mathrm{sec}$ half-space, and the source wavelet used was an $8.0 \mathrm{~Hz}$ peak frequency symmetric wavelet (Fig. 5B). Both the source and receivers were located $1.0 \mathrm{~km}$ above the interface. The Fourier calculation was performed using sample intervals of $62.5 \mathrm{~m}$ in space and $4.687 \mathrm{~ms}$ in time $(p=0.3$ ) for a total of 256 by 128 grid points and 1600 time step iterations. A fourth-order time derivative calculation was used. The Cagniard-de Hoop calculation was obtained using the method described by Aki and Richards (1980), modified for acoustic-wave propagation. Seismograms for the Fourier and Cagniardde Hoop calculations, shown in Figure 8, show good overall agreement between the

\section{Fourier \& Cagniard-DeHoop Sections}

\section{Welded Interface Model (No Free Surface)}
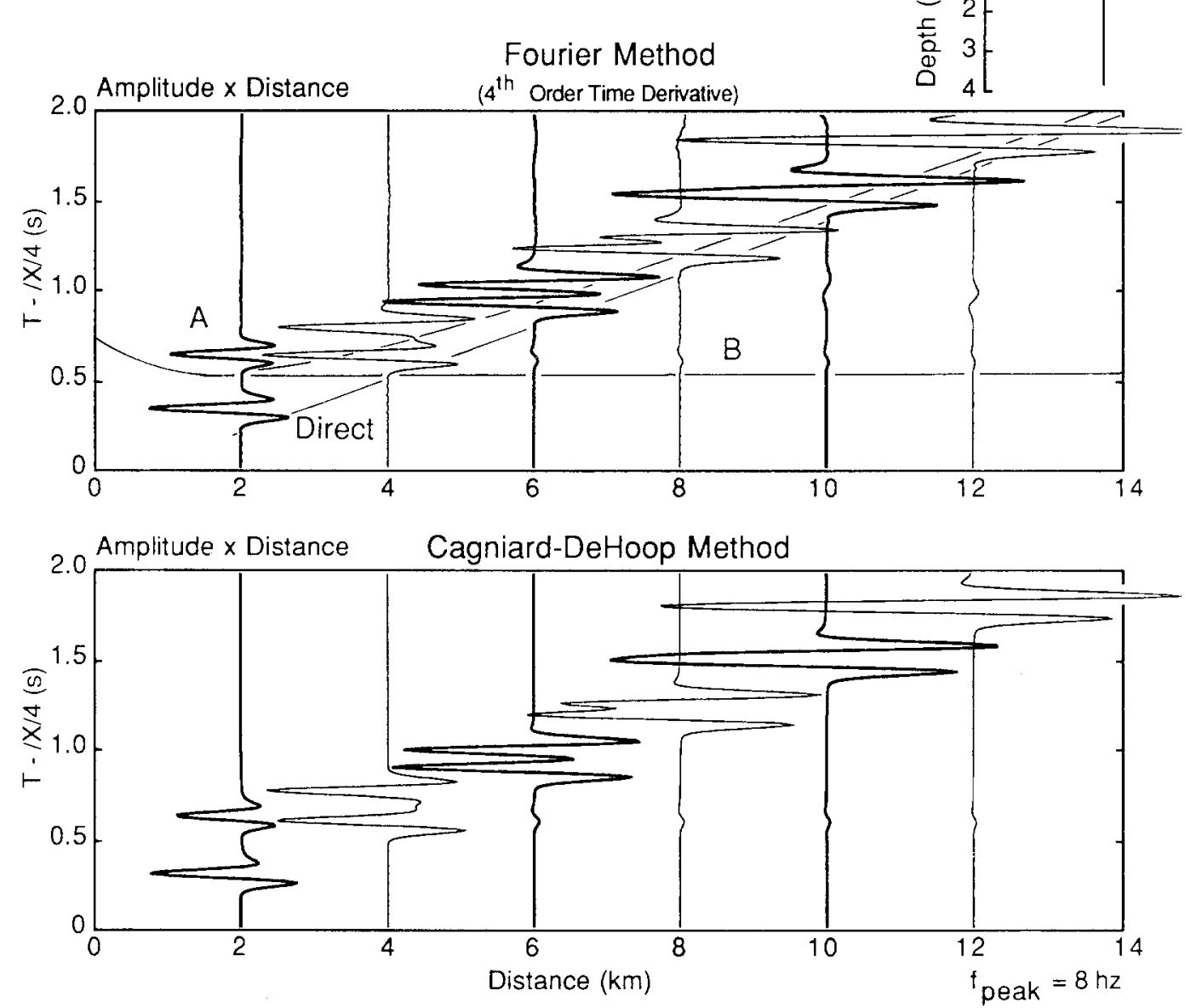

FIG. 8. Seismograms using the Fourier method and the Cagniard-de Hoop method for a model with a single interface. Nonreflecting boundary conditions were used with the Fourier method for all four boundaries. The source function was an $8.0 \mathrm{~Hz}$ peak frequency Ricker wavelet. The Fourier method was implemented with a fourth-order time derivative calculation and sampled at $62.5 \mathrm{~m}$ in space and 4.687 $\mathrm{ms}$ in time using 256 by 128 grid points and 1600 time step iterations. The Cagniard-de Hoop method calculations were based on equations from Aki and Richards (1980, pp. 224-242), which were modified for acoustic-wave propagation. Amplitudes for traces on both the Cagniard-de Hoop and Fourier sections were multiplied times distance for scaling and plotted at a reducing velocity of $4.0 \mathrm{~km} / \mathrm{sec}$. Seismic arrivals include direct, reflected (labeled "A") and refracted (labeled "B") phases. Additionally, the Fourier method shows reflections from artificial boundaries at about 0.9 sec reduced time for traces at 10.0 and $12.0 \mathrm{~km}$. 
two methods for direct, reflected (labeled "A") and refracted (labeled "B") arrivals. A phase advance observed throughout the section for the Fourier method is apparently caused by the manner in which the source function is implemented, as described at the beginning of this section. The Fourier calculation also shows reflections from artificial boundaries at about $0.8 \mathrm{sec}$ reduced time for traces at 10.0 and $12.0 \mathrm{~km}$.

Figure 9 shows a trace by trace comparision for two distances, one near the critical distance and the other at three times the critical distance. In addition to slight phase differences, the Fourier method shows some low amplitude noise in each trace following the refracted arrival. Considering the fundamental differences between Fourier and Cagniard-de Hoop methods, the overall match between seismic traces is encouraging for the Fourier method in simple layered media.

A comparison of the Fourier method was also conducted with the fourth-order

\section{Fourier \& Cagniard-DeHoop Trace Comparison (Welded Interface)}

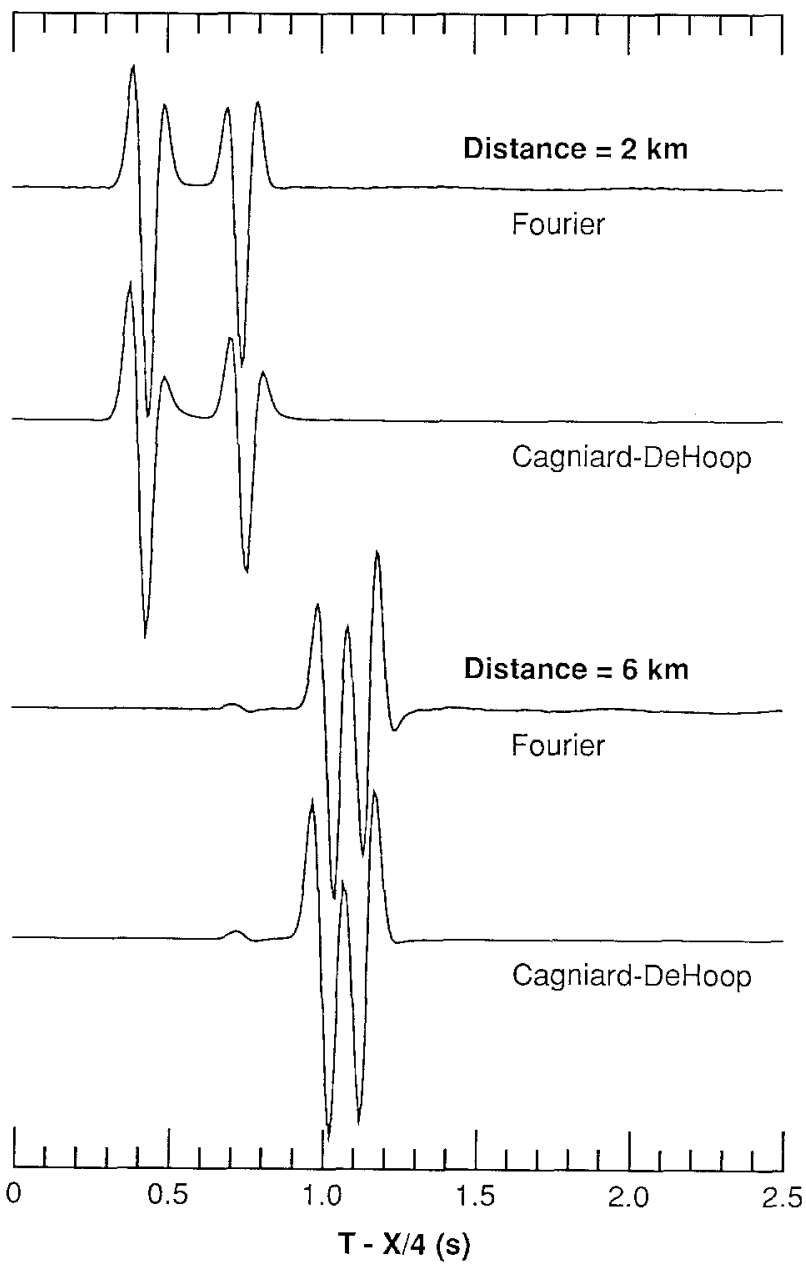

FIG. 9. Trace by trace comparison of sections in Figure 8 for two distances. Trace-normalized amplitudes are compared for Fourier and Cagniard-de Hoop methods for seismograms at about the critical distance $(2.0 \mathrm{~km})$ and three times the critical distance $(6.0 \mathrm{~km})$. Traces reveal a slight phase advance for the Fourier method, but are otherwise remarkably similar. 
implicit and second-order (explicit) finite-difference methods. The velocity model was similar to the welded interface model of Figure 8, except the upper half-space was replaced with a $1.0 \mathrm{~km}$ layer and a free surface. Other model and source parameters remained unchanged. The grid parameters for each method were chosen based on a 2.5 per cent tolerance in group velocity as determined from Figures 2, 3, and 4 . The 2.5 per cent tolerance criteria formed the basis for a somewhat stringent equivalent accuracy comparison between the methods, in that the shortest wavelengths for each method were restricted by the tolerance. Longer wavelengths, which account for most of the amplitude in calculated synthetic traces, were therefore subjected to a much lower tolerance, depending on the shape of the applicable dispersion curve from Figures 2, 3, and 4.

The grid for the second-order finite-difference method was sampled at $12.5 \mathrm{~m}$ in space and $2.188 \mathrm{~ms}$ in time for a total of 1280 by 320 grid points and 3430 time step iterations. The fourth-order finite-difference method used $25.0 \mathrm{~m}$ and $4.375 \mathrm{~ms}$ space and time step intervals respectively for a total of 640 by 160 grid points and 1715 time step iterations. In contrast, the Fourier method, in this case using a second-order time derivative calculation, achieved the same calculation using 256 by 64 grid points and 2400 time step iterations. As an additional comparison, the Fourier method seismograms were also recalculated, sampling the grid and time step sizes twice as dense for a total of 512 by 128 grid points and 4800 time step iterations.

The normal and dense grid Fourier method seismic sections and the finitedifference method seismic sections are shown in Figure 10. Comparison of the four sections reveals differences in phase for later wide-angle and multiple arrivals and variations in amplitude for the head wave. The second-order finite-difference calculation shows more dispersion than the other calculations, in spite of the greater density of grid points. The dispersion is evidenced by both distortion of waveforms and delay of arrivals at far distances. The two finite-difference calculations also show reflections from artificial boundaries at about $0.9 \mathrm{sec}$ reduced time at distances greater than $6.0 \mathrm{~km}$. The artificial reflections were not optimally absorbed and should not be considered for the present comparison.

Figure 11 shows a trace by trace comparison of the Fourier and finite-difference calculations, similar to the Fourier and Cagniard-de Hoop comparison of Figure 9, with traces from near the critical distance and three times the critical distance. The finely sampled Fourier method calculation closely matches the coarse-grid calculation in waveform shape, although some dispersion from the time derivative calculations is evident from the slight phase shifts between the traces. Some amplitude variations are also observed between the two Fourier calculations in secondary phases. Somewhat more noticeable differences exist between the Fourier and finitedifference traces, especially the second-order calculation, primarily in headwave phase and relative amplitudes of later arrivals.

Some of the discrepancies, which are not as apparent in the comparison between Fourier and Cagniard-de Hoop methods of Figures 8 and 9, may partly be due to variations in the source and receiver depth. The source and receivers are located two rows of grid points below the free surface for each calculation (one row for the coarse-grid Fourier calculation). The depth is dependent upon the grid spacing. Apart from the headwave and dispersion-related anomalies, the overall match between the Fourier and finite-difference calculations is close in waveform position, shape and amplitude.

Figure 12 shows a comparison between finite-difference and Fourier methods 


\section{Discrete Grid Sections}

Layer Over Half Space Model
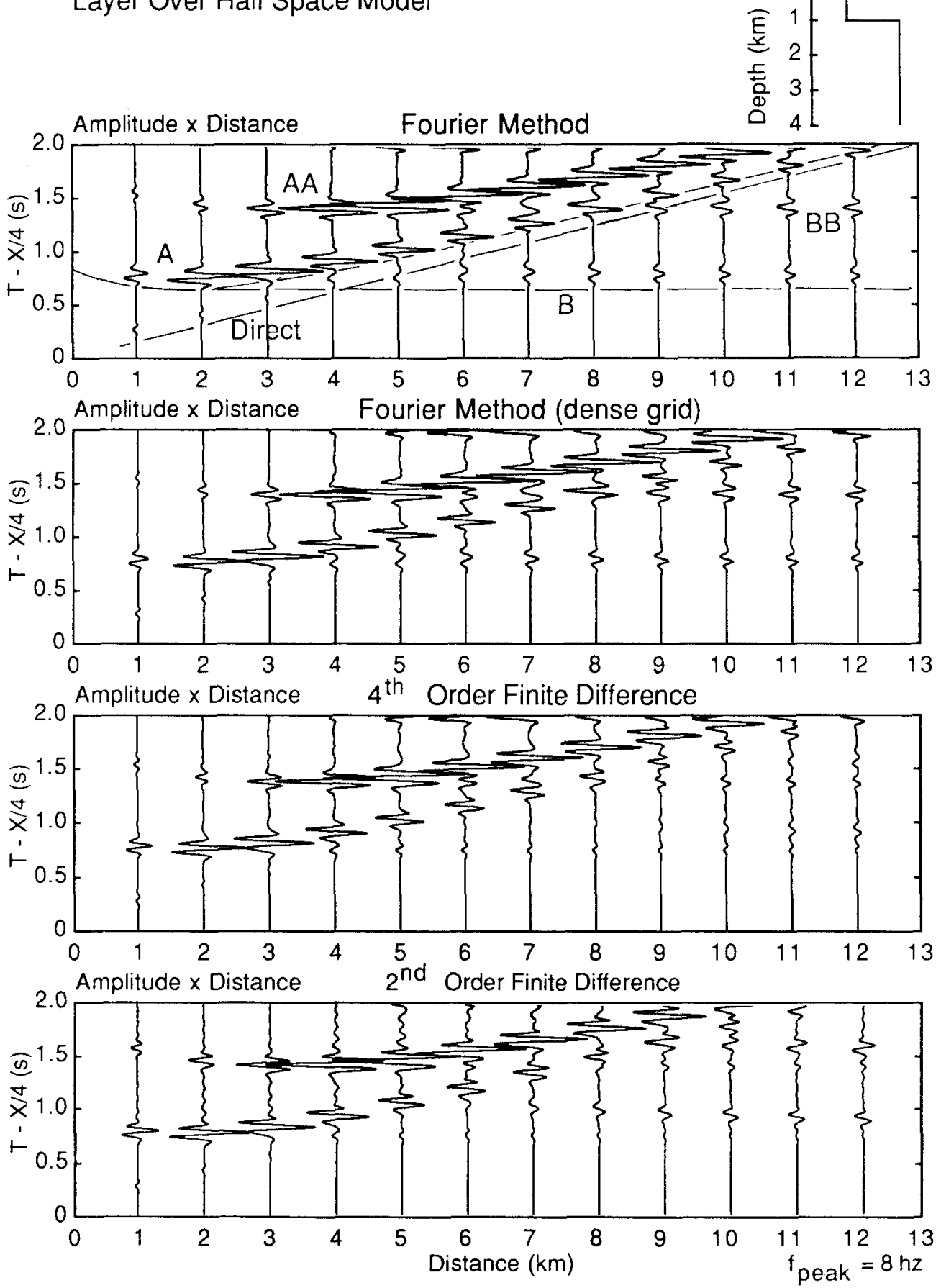

FIG. 10. Seismograms using the Fourier method, a fourth-order finite-difference method and the second-order finite-difference method for a model with a simple layer over a half-space. A free surface boundary condition was used for the top boundary and nonreflecting boundary conditions (Cerjan et al., 1985) for the other three boundaries. The source function was an $8.0 \mathrm{~Hz}$ peak frequency Ricker wavelet. The Fourier method was implemented with a second-order time derivative calculation. The grid for the first Fourier section was sampled at $62.5 \mathrm{~m}$ in space and $3.125 \mathrm{~ms}$ in time using 256 by 64 grid points and 2400 time step iterations. The second Fourier section used halved space and time sample intervals 


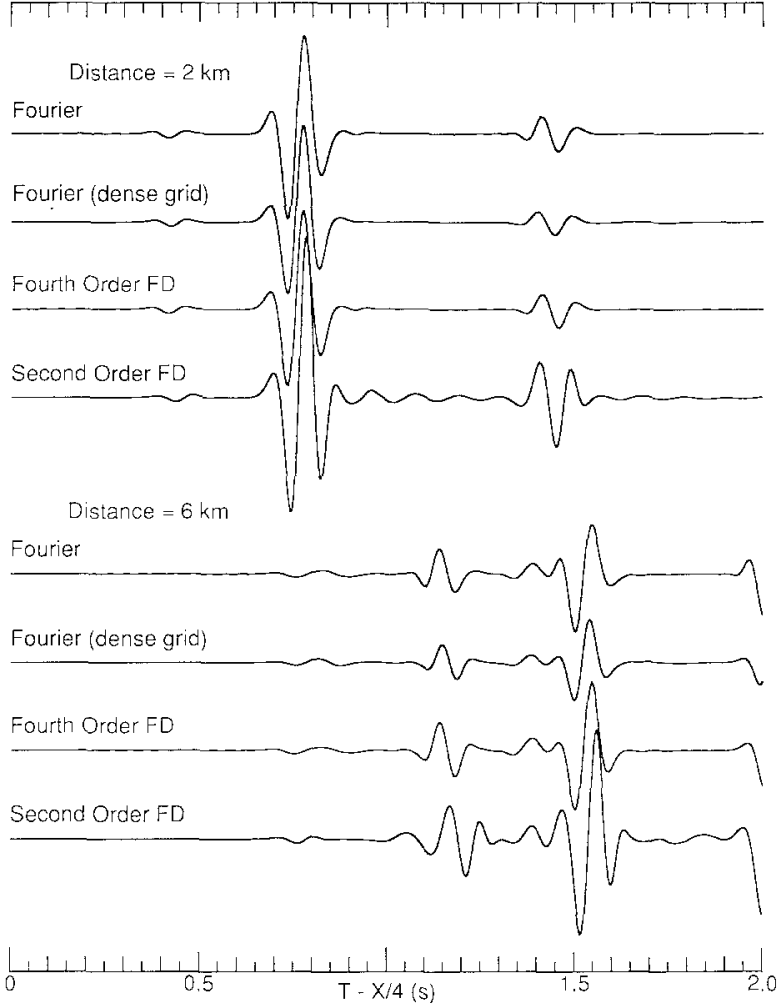

FIG. 11. Trace by trace comparison of sections in Figure 10 for two distances. Traces are compared for Fourier, dense-grid Fourier, fourth-order finite-difference and second-order finite-difference methods for seismograms at about the critical distance $(2.0 \mathrm{~km})$ and three times the critical distance $(6.0 \mathrm{~km})$. Amplitudes are normalized with respect to the first arrival, i.e., the direct wave at $2.0 \mathrm{~km}$ and the head wave at $6.0 \mathrm{~km}$. Traces reveal slight phase discrepancies due to dispersion errors and phase and amplitude differences in the head wave between Fourier and finite-difference methods.

involving CPU time as well as computer storage. Finite-difference (second and fourth-order) and Fourier methods are compared for actual IBM 3083 computer requirements for calculating the model in Figure 10. The height of the hollow vertical bars show the relative CPU time and the height of the solid bars show the relative computer storage requirements for equivalent (two-dimensional) runs. When using a second-order time derivative calculation, the Fourier method required approximately one-third the CPU time of an equivalent second-order finite-difference run. By using a fourth-order time-derivative calculation, the relative CPU time of the Fourier method was reduced to about one fourth. The fourth-order finitedifference method required approximately two thirds the CPU time. With regard to

of $31.25 \mathrm{~m}$ and $1.5625 \mathrm{~ms}$, respectively. The fourth-order finite-difference method was sampled at 25.0 $\mathrm{m}$ in space and $4.375 \mathrm{~ms}$ in time using 640 by 160 grid points and 1715 time step iterations. The secondorder finite-difference method was sampled at $12.5 \mathrm{~m}$ in space and $2.1875 \mathrm{~ms}$ in time using 1280 by 320 grid points and 3430 time step iterations. Amplitudes for traces in all three sections were multiplied times distance for scaling and plotted at a reducing velocity of $4.0 \mathrm{~km} / \mathrm{sec}$. Seismic arrivals include direct, reflected (labeled "A"), refracted ("B") and multiply reflected and refracted ("AA" and "BB", respectively) phases. Reflections from artificial boundaries are also present at about 0.9 sec reduced time for distances greater than $6.0 \mathrm{~km}$. 


\section{CPU Time and Storage Comparisons}

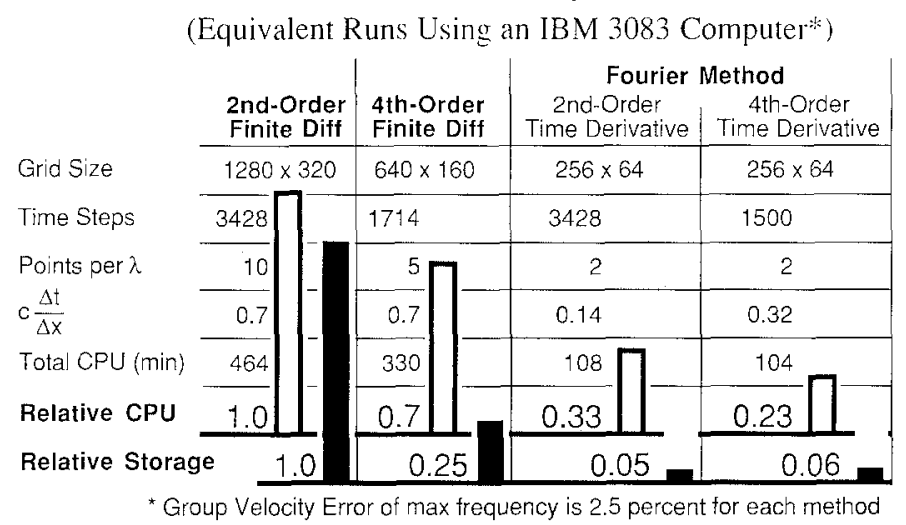

Fig. 12. Actual CPU time and storage comparisons for second-order explicit, fourth-order implicit and Fourier methods for equivalent calculations of the two-dimensional layer over half-space model in Figure 10. The parameters for each method were determined from the group velocity curves of Figures 2,3 , and 4 by arbitrarily using a group velocity error of 2.5 per cent for the maximum frequency (three times $8.0 \mathrm{~Hz}$ ) for each method. The Fourier method calculations were performed using both a secondand fourth-order time derivative calculation. Computations were conducted on an IBM 3083 computer without using vector processing or virtual memory. The explicit time requirements were extrapolated from calculating and timing several time step iterations. Fourth-order and Fourier CPU times were recorded for calculations of Figure 10. The Fourier method calculations required about one-third or onefourth the CPU time of the second-order explicit calculations, as shown by the height of the hollow vertical bar, depending which time derivative calculation method was used. The Fourier method required less computer storage by nearly a factor of 20 .

computer storage, the fourth-order finite-difference method required a factor of 4 less computer storage than the second-order method. The storage requirements for the Fourier method was less by nearly a factor of 20 . These efficiency factors, which apply to two-dimensional models, are not quite as dramatic as those stated by Fornberg (1987), who suggested a factor of 256 fewer grid points for the Fourier method. The stringent group velocity tolerance described previously and used by the calculations account for the somewhat more modest efficiency factors stated here, which are nevertheless significant. For three-dimensional models, the efficiency factors become even more significant and dramatic.

The calculations shown above are for a simple model characterized by depth variations only. The primary advantage of discrete grid methods such as Fourier and finite-difference methods, howeever, is their ability to perform calculations on complicated laterally varying models. Figure 13 shows a layer over half-space similar to the velocity model for Figure 10, modified to include heterogeneities. The Fourier method calculation used the same model and source parameters as Figure 10 except that the lower half-space consisted of random velocity variations of up to 10 per cent. The variations in velocity were produced using a uniform distribution of random numbers and bandpass-filtered in two dimensions. The average wavelength of velocity variations after filtering was $0.5 \mathrm{~km}$, or one seismic wavelength for the peak source energy. The importance of understanding random velocity variations is discussed by Frankel and Clayton (1986), who investigated scattering effects on amplitudes, travel times, spectra, and waveforms for short-period seismic waves (less than $1 \mathrm{sec}$ ). They used a fourth-order finite-difference method to show that scattering produced by such velocity variations can explain both travel-time anomalies reported for teleseismic arrivals across large scale seismic arrays (e.g., LASA 


\section{Heterogeneous Model Section}

Layer Over Random Velocity Half Space Model

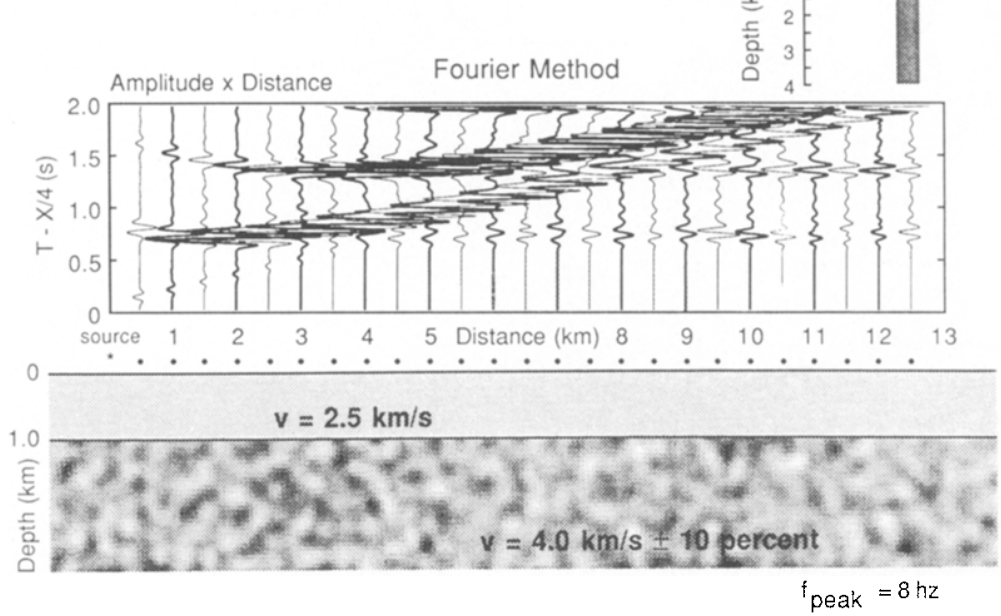

FIG. 13. Seismograms for model with random velocity variations in the basement using the Fourier method. The model and calculation parameters are identical to Figure 10 except for velocity variations of up to \pm 10 per cent in the lower half-space. The velocity model for the half-space was formed using a uniform distribution random number generator and bandpass-filtered in both spatial dimensions passing wavelengths on the order of the $8.0 \mathrm{~Hz}$ source function. The arrow indicates the head wave of varying amplitude, which distinguishes this seismic section from the Fourier sections of Figure 10.

and NORSAR) and the presence of seismic coda at frequencies above $30 \mathrm{~Hz}$ commonly observed in microearthquake waveforms.

The wide-angle reflection and multiple reverberations in Figure 13 can be seen as well as the horizontal head wave and reverberation head waves. The important difference between calculations for Figures 10 and 13 is the variation of amplitudes along the wide-angle reflection and particularly the head wave indicated by the arrow in the latter figure. Complicated lateral heterogeneous models such as Figure 13 can only be modeled using discrete grid methods such as finite-difference and Fourier methods. Detailed testing of the Fourier method for such models, which is beyond the scope of the present paper, is therefore restricted to comparison with other discrete grid methods.

\section{CONCLUSIONS}

The Fourier method and a fourth-order implicit finite-difference method for calculating synthetic seismograms in acoustic media are in many instances, including certain heterogeneous models, capable of performing calculations equivalent in accuracy to the second-order explicit finite-difference method using better than one-fourth and two-thirds the CPU time and using one-twentieth and one-fourth the computer storage, respectively. The seismogram errors for each of the discrete grid methods resulting from numerical dispersion can be predicted analytically by calculating both phase and group velocity curves, and utilizing group velocity calculations to determine errors in travel time as a function of frequency. Both phase and group velocity curves were calculated for all three methods, from which criteria were established for estimating equivalent accuracy of each method. The criteria used for the comparisons in this paper was stringent, a 2.5 per cent tolerance in group velocity for the shortest wavelength propagated. Conceivably, the efficiency factors quoted above for CPU time and storage improvement could be even greater 
without seriously degrading the accuracy of the calculations for wavelengths of significant amplitude. Comparisons of the Fourier method with the Cagniard-de Hoop method confirmed that the Fourier method was capable of performing equivalent calculations for simple layered media.

The primary advantage of the coarse grid methods are their ability to model relatively large, laterally heterogeneous media, ultimately including three-dimensional variations in structure and even anisotropic features. Equally important is their ability to model complicated or rapidly varying velocity structures such as those in Figure 13. The Fourier method, in particular, has become a valuable tool in modeling velocity structures of deeper and longer extent than feasible with traditional finite-difference techniques.

\section{ACKNOWLEDGMENTS}

We wish to thank Kenneth H. Olsen of Los Alamos National Laboratory and William J. Lutter of Purdue University for their constructive ideas. This research was funded by NSF grants EAR- 8418672 (LWB and CRD) and EAR-8518147 (RLN).

\section{REFERENCES}

Aki, K. and P. G. Richards (1980). Quantitative Seismology: Theory and Methods. W. H. Freeman and Company, San Francisco, 557 p.

Alford, R. M., K. R. Kelly, and D. M. Boore (1974). Accuracy of finite-difference modeling of the acoustic wave equation, Geophysics $\mathbf{3 9}, 834-842$.

Bayliss, A., K. E. Jordan, B. J. LeMesurier, and E. Turkel (1986). A fourth-order accurate finitedifference scheme for the computation of elastic waves, Bull. Seism. Soc. Am. 76, 1115-1132.

Cerjan, C., K. Kosloff, R. Kosloff, and M. Reshef (1985). A nonreflecting boundary condition for discrete acoustic-wave and elastic-wave equations, Geophysics 50, 705-708.

Dablain, M. A. (1986). The application of high-order differencing to the scalar wave equation, Geophysics 51, 54-56.

Daudt, C. R. (1983). Finite Difference Synthetic Seismogram Calculations Utilizing Acoustic Models and Explicit and Implicit Formulations, M.S. Thesis, Purdue University, West Lafayette, Indiana.

Emerman, S. H., W. Schmidt, and R. A. Stephen (1982). An implicit finite difference formulation of the elastic wave equation, Geophysics 47, 1521-1526.

Fairweather, G. and A. R. Mitchell (1965). A high accuracy alternating direction method for the wave equation, J. Inst. Maths Applics. 1, 309-316.

Fornberg, B. (1987). The pseudospectral method: comparisons with finite differences for the elastic wave equation, Geophysics 52, 483-501.

Fornberg, B. (1988). The pseudospectral method: accurate representation of interfaces in elastic wave calculations, Geophysics 53, 625-637.

Frankel, A. and R. W. Clayton (1986). Finite difference simulations of seismic scattering: implications for the propagation of short-period seismic waves in the crust and models of crustal heterogeneity, J. Geophys. Res. 91, 6465-6489.

Johnson, O. G. (1984). Three-dimensional wave equation computations on vector computers, Proc. IEEE 72, 90-95.

Kosloff, D. D. and E. Baysal (1982). Forward modeling by a Fourier method, Geophysics 47, 1402-1412.

Kosloff, D. D., M. Reshef, and D. Lowenthal (1984). Elastic wave calculations by the Fourier method, Bull. Seism. Soc. Am. 74, 875-891.

Marfurt, K. J. (1984). Accuracy of finite-difference and finite-element modeling of the scalar and elastic wave equations, Geophysics 49, 533-549.

McKee, S. (1973). High accuracy A. D. I. methods for hyperbolic equations with varible coefficients, $J$. Inst. Maths Applics. 11, 105-109.

Reshef, M. and D. D. Kosloff (1985). Applications of elastic forward modeling to seismic interpretation, Geophysics 50, 1266-1272.

Reshef, M., D. Kosloff, M. Edwards, and C. Hsiung (1988a). Three-dimensional acoustic modeling by the Fourier method, Geophysics 53, 1175-1183.

Reshef, M., D. Kosloff, M. Edwards, and C. Hsuing (1988b). Three-dimensional elastic modeling by the Fourier method, Geophysics 53, 1184-1193. 
Ricker, N. H. (1977). Transient Waves in Visco-Elastic Media, Elsevier, Amsterdam, 278 pp.

Sochacki, J. R. Kubichek, J. George, W. R. Fletcher, and S. Smithson (1987). Absorbing boundary conditions and surface waves, Geophysics 52, 60-71.

Tadmor, E. (1986). The exponential accuracy of Fourier and Chebyshev differencing methods, SIAM J. Numer. Anal. 23, 1-10.

Tal-ezer, H. (1986). Spectral method in time for hyperbolic equations, SIAM J. Numer. Anal. 23, 1126.

Trefethen, L. N. (1982). Group velocity in finite difference schemes, SIAM Review 24, 113-136.

Department of EARTh and ATMOSPheric Sciences

Purdue University

WEST LAFAYETTE, INDIANA 47907

Manuscript received 9 June 1988 